\title{
Optimization of Hydraulic Retarder Based on CFD Technology
}

\author{
Li Hao ${ }^{1, a^{*}}$, Ren Xiaohui ${ }^{1, b}$ \\ ${ }^{1}$ College of Vehicle and Energy, Yanshan University, Qinhuangdao, China \\ alihao@ysu.edu.cn, b758649793@qq.com
}

\begin{abstract}
Keywords: hydraulic retarder; numerical calculation; flow field characteristics; vane angle.
Abstract. The three-dimensional geometric model of hydraulic retarder is built based on an enterprise production as reference prototype, On CFD platform, based on the three-dimensional entity model of the hydraulic retarder, the internal flow field at full-filling condition is simulated and the braking performance is calculated. The simulation results show that there were some problems such as vast vortexes and wall flow separations which added braking torque losses. In order to improve the braking torque, this paper built some schemes with different vane degrees from $30^{\circ}$ to $60^{\circ}$, after calculating and simulating, got the best optimization scheme is $45^{\circ}$. The simulation result of optimization schemes and prototypes shows that the braking performance of hydraulic retarder is obviously improved.
\end{abstract}

\section{Introduction}

Hydraulic retarder, as a kind of auxiliary brake, is applied to stabilize vehicle speed in long slope road. Overload brake is common for heavy duty truck, driving in mountainous areas and mining road. It is hard to meet people's braking requirement only rely on the traditional brake system. The hydraulic retarder is widely applied in heavy vehicle due to it has high and continuous braking performance with a compact structure[1].A retarder is usually consists of rotor, stator, cooling system and control system, the retarder always locates between convert and transmission. During the working rotating mechanical energy is transferred into heat energy and the heat is brought to outside atmosphere by cooling system, at the same time the brake torque is generated and work at tires to slow down the vehicle speed[2].The structure parameter of hydraulic retarder is an important factor that effect the braking performance[3].

In this paper, the three-dimensional geometric model of hydraulic retarder is built based on an enterprise production as reference prototype, On CFD platform, the internal flow is simulated and the influence of blade angle on the internal flow is analyzed. In order to improve the brake performance, put out some different optimization schemes of the blade angle.

\section{Simulation of Prototype}

Condition Hypothesis. Internal flow field is relatively complex. The following assumptions are made for the convenience of calculation[4]. According to the requirements of SAE standards of hydraulic transmission oil, the density see as constant for the oil density and viscosity change is very small when retarder works. The density and viscosity is respectively defined as $\rho=860 \mathrm{~kg} / \mathrm{m}^{3}$ and $\mu=0.006 \mathrm{~kg} / \mathrm{m} \cdot \mathrm{s}$, the oil can be regarded as incompressible viscous fluid. The influence of the temperature for flow field can be ignored due to the cooling system keeps working. Rotor and stator have no axial displacement and the structure has no deformation. When the hydraulic retarder is working as normal, the oil flow rate of cooling system is less than $0.2 \%$ of the circulating flow rate. The oil leakage is ignored for it is far less than circulation flow when retarder works [5].

Model of Hydraulic Retarder. Enterprise production is used in this paper as reference prototype. The rotor and stator effective circular circle diameters are $363 \mathrm{~mm}$, leaf number is 36 and 32 respectively. Considering the actual structure of the hydraulic retarder, this paper extracted the whole flow space as computational domain by whole flow passage form [6]. The whole calculation basin is divided into strong adaptability of hybrid grid by unstructured grid generation technique [7]. 
Simulation Result Analyzing of Prototype. The distribution characteristics of internal special flow surface is analyzed under the speed of $n_{B}=1200 \mathrm{r} / \mathrm{min}$ and $n_{B}=1600 \mathrm{r} / \mathrm{min}$. The flow channel vertical profile is established in computational domain after convergence of iterative calculation. The axial surface velocity vector distribution is shown in Figure 1 . The velocity vector of internal circle section trends circular flow obviously. The flow speed in the rotor basin from import to export increases gradually and reaches the maximum value near the outer ring; In the stator basin from import to export the flow velocity is gradually decreasing, the minimum value appears at the exit.

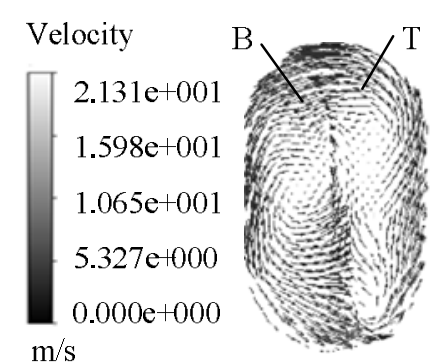

a) $\mathrm{n}_{\mathrm{B}}=1200 \mathrm{r} / \mathrm{min}$

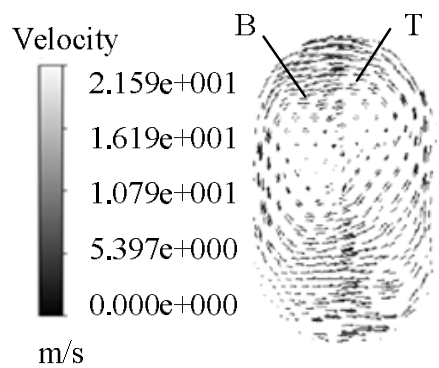

b) $\mathrm{n}_{\mathrm{B}}=1600 \mathrm{r} / \mathrm{min}$

Figure 1. Axial velocity vector distribution

The velocity vector distribution of the model chord sections under different rotating speeds shows in Figure2. Vortex occurs in the stator basin and it intensifies with the rotor speed's increasing, that has adverse effect on the braking torque output. After analyzing got that the blade angle has a great impact on the vortex's formation and velocity vector's direction. So that this paper proposed the correction scheme of blade angle to make the flow field velocity distribution smoother and the braking torque higher.

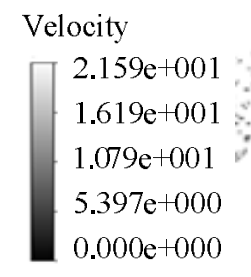

$\mathrm{m} / \mathrm{s}$

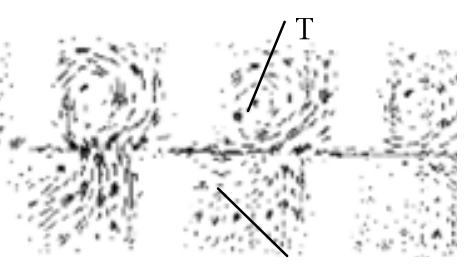

$\mathrm{B}$

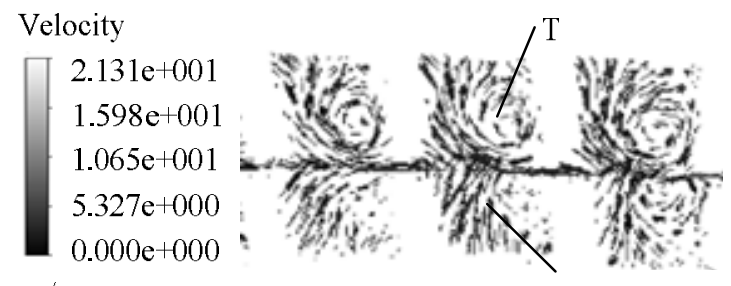

$\mathrm{m} / \mathrm{s}$

a) $n B=1200 \mathrm{r} / \mathrm{min}$
Figure 2 . Velocity vector distribution on the part of chord sections

\section{Optimization of Blade Angle}

After analyzing the flow field distribution of prototype, got that unreasonable blade angle is the major cause lead to the appearance of vortex at chord section, and the shape of circular circle has little effect on the simulation. In order to improve the braking performance, form of the blade structure was also taken [8], in this paper has optimized the prototype's blade angle without changing the size of the circular circle.

Evaluation Index of Blade Structure Parameters. When hydraulic retarders have similar flow channel geometry, under the same conditions their braking moment coefficient $\lambda_{m}$ are equal. $\lambda_{m}$ can response the stand or fall of braking performance, it can be used as the evaluation index of blade structure parameters' optimizing[9].

$T=\lambda_{m} \rho g n_{B}^{2} D^{5}$

Where $\mathrm{T}$ is braking torque $\left(\mathrm{kg} / \mathrm{m}^{3}\right), \lambda_{\mathrm{m}}$ is braking moment coefficient $\left(\mathrm{min}^{2} / \mathrm{r}^{2} \cdot \mathrm{m}\right), \rho$ is oil's density $\left(\mathrm{m} / \mathrm{s}^{2}\right)$, $\mathrm{g}$ is gravitational acceleration $\left(\mathrm{m} / \mathrm{s}^{2}\right), \mathrm{n}_{\mathrm{B}}$ is rotor $\operatorname{speed}(\mathrm{r} / \mathrm{min})$, $\mathrm{D}$ is effective diameter of circular circle $(\mathrm{m})$.

By the above formula known, without changing the size of effective diameter of circular circle, when the rotor speed is keeping, hydraulic retarder's braking torque is proportional to the coefficient $\lambda_{\mathrm{m}}$, so that the braking torque also can be used as the evaluation index of blade structure parameters' optimizing 
Numerical Simulation of Internal Flow Field of Different Blade Angle Schemes. According to related information [10], the blade angle always choose from $30^{\circ}$ to $60^{\circ}$, based on the sensitivity analysis of the blade forward angle on braking performance under the streamline theory, and reference for selection experience in the structural design of hydraulic retarder blade angle, this paper has chosen different blade forward angles between $30^{\circ}$ and $60^{\circ}$, then built different geometric models without changing circular circle structure size.

Take the numerical simulation of internal flow field of different blade angle schemes under the condition of $n_{B}=1200 \mathrm{r} / \mathrm{min}$ and full-filling. The calculated braking torque value is shown in Figure 3 .

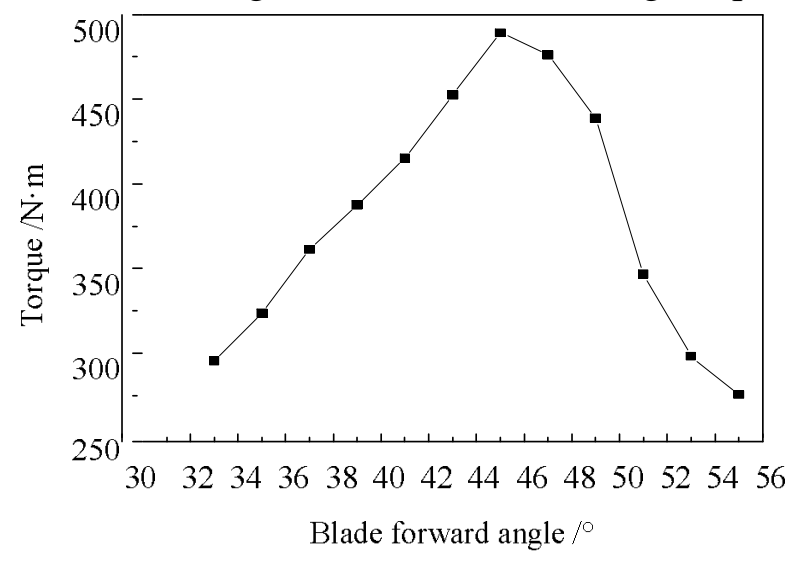

Figure 3. Blade forward angle - torque curve

The Figure 3 shows that, with the blade angle increasing, the change trend of brake torque of hydraulic retarder is first increases and then decreases. This result is consistent with the analysis under streamline theory. The simulation shows that when the blade angle is $45^{\circ}$ the braking torque is the highest

Simulation Result Analyzing of Three Selected Schemes. Select three schemes of $43^{\circ}, 45^{\circ}$, and $47^{\circ}$, comparative analysis on the internal flow field distribution. The relative velocity vector distribution on the part of model chord sections is shown in Figure 4.

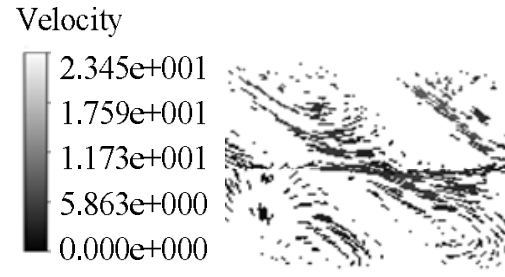

$\mathrm{m} / \mathrm{s}$
Velocity

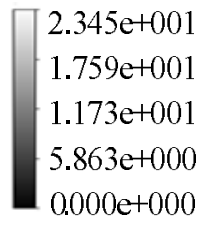

$\mathrm{m} / \mathrm{s}$

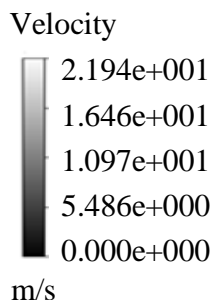

$\mathrm{m} / \mathrm{s}$

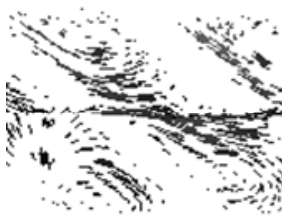

b) $45^{\circ}$

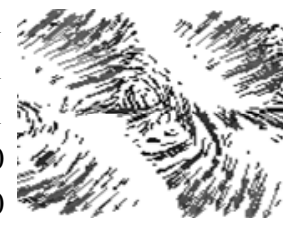

c) $47^{\circ}$

Figure 4. Velocity vector distribution on the part of moddle chord Sections of three schemes

The static pressure distribution on different chord plane in the $45^{\circ}$ optimization scheme is shown in Figure 5.
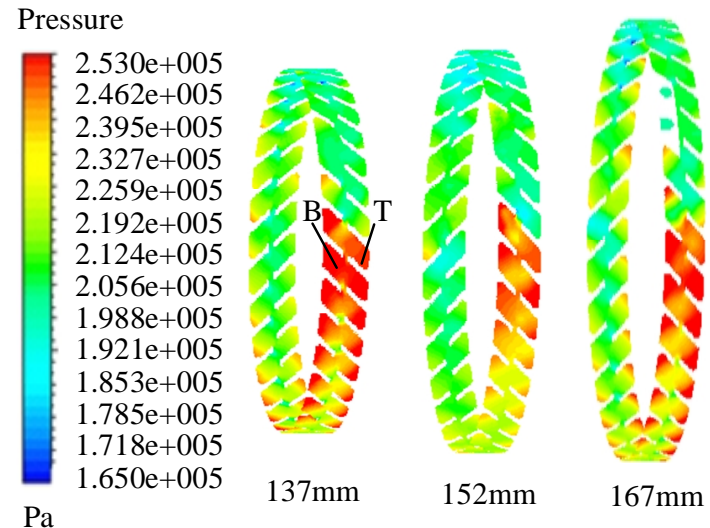

Figure 5. Static pressure distribution on $45^{\circ}$ chord plane in the three optimization schemes 
The figure shows that the pressure is all presented lamellar distribution on the different chord plane in the three schemes, between the pressure surface of stator vane and the outer ring the movement direction of high-speed flow suddenly turn, due to the impact of flow movement has resulted in the pressure value at the turning point is higher, and the pressure of suction surface at the vane leading edge is lower. When the blade angle of retarder is $45^{\circ}$, the high pressure distribution area of chord plane flow field is the biggest. It shows that local pressure value at this time is highest.

The pressure distribution on the cross section which is near the interface between stator and rotor and the direction is perpendicular to the axis of rotation is shown in Figure 6.
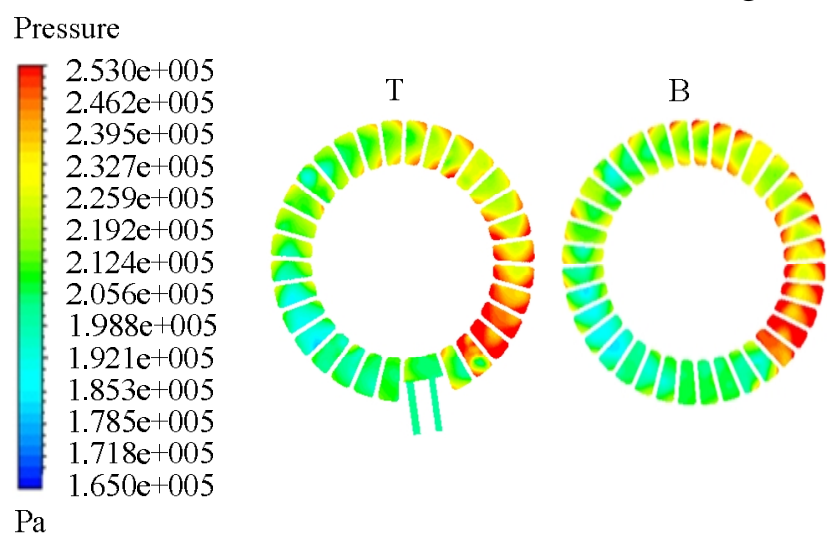

Figure 6. Pressure distribution on the cross section of the $45^{\circ}$ optimization scheme

A vortex motion in the unit is produced by the transverse differential pressure between pressure surface and suction surface, the vortex motion increases with the increase of transverse differential pressure. The braking torque is increased too.

The Figure VII shows that, the transverse differential pressure of the flow field motion is largest in the $45^{\circ}$ scheme. It is more favorable to the braking torque at this time.

After a series of considerations, it takes $45^{\circ}$ anteverted angle as the best solution for hydraulic retarder blade angle optimization ultimately. These considerations include comparative analysis of flow field distribution of the three blade angle schemes under a specific speed, analysis of hydraulic retarder braking torque calculation results of different blade angle schemes under specific speed, at the same time, it takes the effect of blade angle on the strength and the blade production convenience into account. In this scheme, braking torque value produced by hydraulic retarder is the largest and optimization effect is the best.

\section{Braking Performance Comparison}

After internal flow field numerical simulation of $45^{\circ}$ optimization scheme under the whole filling condition and different rotational speeds, the calculated braking torque values at each speed are shown in Table 1.

Table1. Brake Torque values of $45^{\circ}$ Optimization in the Whole Filling Condition

\begin{tabular}{|l|l|l|l|l|l|}
\hline $\mathrm{nB}(\mathrm{r} / \mathrm{min})$ & 800 & 1000 & 1200 & 1400 & 1600 \\
\hline $\mathrm{T}(\mathrm{N} . \mathrm{m})$ & 205.5 & 329.5 & 489.3 & 657.8 & 911.5 \\
\hline
\end{tabular}




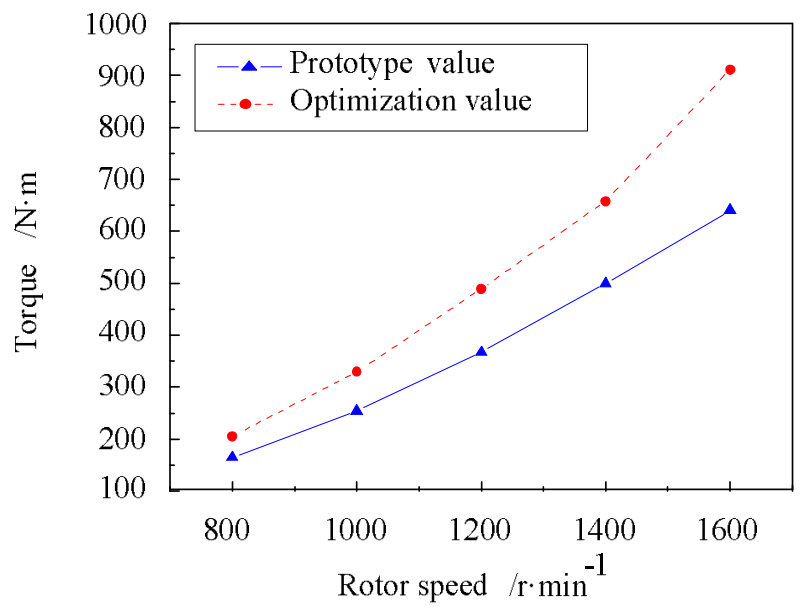

Figure 7. simulation results of original prototype and optimization

Combined with the torque calculation results of original prototype under the same working conditions, a speed - torque curve shown in Figure 7 is fitted out in the same coordinate system. Comparative analysis shows that, without changing circular circle structure size, brake torque value produced by the hydraulic retarder with the $45^{\circ}$ anteverted blade angle is greater than which generated by the original prototype under the same conditions, the brake torque value is up by more than $24 \%$ after optimizing. The results show that changing the blade forward angle has a great influence on the braking torque generation of hydraulic retarder.

\section{Conclusion}

Sensitivity analysis of the blade forward angle on braking performance under the streamline theory shows that the optimal value of the anteverted blade angle exists.

Determining $45^{\circ}$ blade forward angle as the best optimization scheme based on the analysis of hydraulic retarder braking torque calculation results of different blade angle schemes.

Optimized braking torque values under different speeds are increased by more than $24 \%$, which indicates that to optimize the blade angle will help to improve the brake performance of hydraulic retarder, and can provide theoretical basis for optimizing design of blade structure of hydraulic retarder.

\section{References}

[1] Wei W, Yan Q D. Study on Hydrodynamic Torque Converter Parameter Integrated Optimization Design System Based on Tri-Dimensional Flow Field Theory[J]. SAE 2008-01-1525.

[2] Wu Chao, Xu Ming, Li Huiyuan,et al. Analysis of Characteristic and Development of Vehicle Hydraulic Retarder[J]. Vehicle and Power Technology, 2011(01): 51-55.

[3] Xie Rong, Shan Yujiao, Wang X iaofang. Numerial Simulation on Flow Performance and Blade Profile Optimal Design of Mixed-flow Pump Impeller[J]. Journal of Drainage and Irrigation Machinery Engineering, 2010, 28(4): 296-299.

[4] Wang Tie. Research on Simulation Method for Analysis on Tree-Dimension Flow Field of Automotive Hydraulic Retarder [D]. Changchun: Jilin University, 2007: 39-40.

[5] Flack R, Brun K. Fundamental Analysis of the Secondary Flows and Jet-Wake in a Torque Converter Pump--Partl : Model and Flow in a Rotating Passage[J]. ASME Journal of Fluids Engineering, 2005, 127: 66-74.

[6] Shi X Z, Liu B, Zhang Z, Measures of Internal Flow Calculation Accuracy Improvement on Hydraulic Torque Converter[J]. Procedia Engineering, 2012, 28: 235-240. 
[7] Yang T, Liang R L,Guo X X, et al. Strength Analysis and Modal Analysis of Hydraulic Retarder[J]. SAE Paper 2009-01-2896.

[8] Wang Feng. Study on the Braking Performance of the Hydraulic Retarder Based on Flow Field Analysis [D]. Beijing: Beijing Institute of Technology, 2007: 20-33.

[9] Li Xuesong, Liu Chunbao, Cheng Xiusheng, et al. Blade Profile Optimal Desige of Impeller Besed on Flow Performance [J]. Transactions of the Chinese Society for Agricultural Machinery, 2014, 45(6): 21

[10] Yu Guobao. Research on the Structural Parameter Optimization of Heavy Vehicle and Hydraulic Retarder [D]. Nanjing: Nanjing Institute of Technology, 2013: 7, 55-56 